\title{
Gaussian Weighted Projection for Visualization of Cardiac Calcification
}

\author{
Xiang Chen; Ke Li, Robert Gilkeson; Baowei Fei
}

\begin{abstract}
At our institution, we are using dual-energy digital radiography (DEDR) as a cost-effective screening tool for the detection of cardiac calcification. We are evaluating DEDR using CT as the gold standard. We are developing image projection methods for the generation of digitally reconstructed radiography (DRR) from CT image volumes. Traditional visualization methods include maximum intensity projection (MIP) and average-based projection (AVG) that have difficulty to show cardiac calcification. Furthermore, MIP can over estimate the calcified lesion as it displays the maximum intensity along the projection rays regardless of tissue types. For AVG projection, the calcified tissue is usually overlapped with bone, lung and mediastinum. In order to improve the visualization of calcification on DRR images, we developed a Gaussian-weighted projection method for this particular application. We assume that the CT intensity values of calcified tissues have a Gaussian distribution. We then use multiple Gaussian functions to fit the intensity histogram. Based on the mean and standard deviation parameters, we incorporate a Gaussian weighted function into the perspective projection and display the calcification exclusively. Our digital and physical phantom studies show that the new projection method can display tissues selectively. In addition, clinical images show that the Gaussianweighted projection method better visualizes cardiac calcification than either the AVG or MIP method and can be used to evaluate DEDR as a screening tool for the detection of coronary artery diseases.
\end{abstract}

Keywords: Visualization, perspective projection, computed tomography (CT), coronary artery disease (CADs)

\section{INTRODUCTION}

Cardiovascular disease is the leading cause of death in the United States [1]. The relationship between coronary artery calcification and atherosclerotic heart diseases has been well documented [2, 3]. Early detection of cardiac calcification is directly helpful for diagnosing heart diseases. Computed tomography (CT) is an established tool for detecting cardiac calcification. However, the increasing incidence of cardiovascular diseases requires a cost-effective, accurate, and noninvasive screening technique for large-scale populations. At our institution, we are using dual-energy digital radiography (DEDR) as a cost-effective screening tool for the detection of cardiac calcification $[4,5,6,7,8]$. We are evaluating DEDR using CT as the gold standard $[9,10]$. We are developing image projection methods for the generation of digitally reconstructed radiography (DRR) from CT image volumes. Unfortunately, it is difficult to visualize calcification using traditional projection methods such as maximum intensity projection (MIP) and average-based projection (AVG) because cardiac calcification is overlapped with bone, lung and mediastinum in space and because the calcium's volume is less than $1 \%$ of the heart. Furthermore, MIP can over estimate the calcified lesion as it displays the maximum intensity along the projection rays regardless of tissue types. In order to improve the visualization of calcification on DRR images, new visualization methods are needed for this particular application.

\footnotetext{
* Corresponding authors: Department of Radiology, Case Western Reserve University, 11100 Euclid Ave, Cleveland, Ohio 44106. Phone: 216-844-5281, Fax: 216-844-3106, E-mail: Baowei.Fei@case.edu. XC is with Case Western Reserve University and Xi'an Jiaotong University. KL is with Case Western Reserve University. RG is with University Hospitals Case Medical Center. FB is with Case Western Reserve University.
}

Medical Imaging 2008: Visualization, Image-guided Procedures, and Modeling, edited by

Michael I. Miga, Kevin Robert Cleary, Proc. of SPIE Vol. 6918, 691831, (2008)

$1605-7422 / 08 / \$ 18 \cdot$ doi: $10.1117 / 12.772597$

Proc. of SPIE Vol. 6918 691831-1 


\section{METHODS FOR PROJECTION}

We developed a Gaussian-weighted projection (GWP) method based on the shear-warp factorization perspective projection method. We assume that the CT intensity values of calcified tissues have a Gaussian distribution. We use multiple Gaussian functions to fit the intensity histogram. Based on the mean and standard deviation parameters, we incorporate a Gaussian weighted function into the perspective projection and display the calcification exclusively. We applied digital phantom, physical phantom and clinical data sets to evaluate the projection method.

\subsection{Shear-warp Factorization Method}

Our projection methods for DRR images are based on a shear-warp factorization method [11]. In this method, a viewing transformation is applied to simplify the projection processing. The algorithm uses a principal viewing axis to choose a set of CT voxel slices to be resampled for composition. It also determines the order of the slices along the front-to-back direction of the image volume.

Generally, a perspective viewing transformation matrix $\mathrm{M}_{\text {view }}$ includes a permutation $\mathrm{P}$, a shift of the origin $T_{\text {shift }}$, a 3D perspective shear scale $M_{\text {shear }}$, and a $2 \mathrm{D}$ warp $M_{\text {warp }}$ as: $M_{\text {view }}=M_{\text {warp }} \cdot M_{\text {shear }} \cdot T_{\text {shift }} \cdot P$, where $M_{\text {view }}$ is a $4 \times 4$ affine viewing transformation matrix that transforms points from the object space to the image space. $M_{\text {view }}$ will be simplified as: $M_{\text {view }}=M_{\text {warp }} \cdot M_{\text {shear }}$, where $M_{\text {shear }}$ is the shear transformation, a product of a pure shear transformation $M_{s h}$ and a perspective scaling transformation $M_{s}$, i.e., $M_{\text {shear }}=M_{s} \cdot M_{s h}$. The pure shear transformation $\mathrm{M}_{\mathrm{sh}}$ is represented as:

$$
\mathrm{M}_{\mathrm{sh}}=\left[\begin{array}{cccc}
1 & 0 & -\frac{\mathrm{e}_{x}}{\mathrm{e}_{z}} & 0 \\
0 & 1 & -\frac{\mathrm{e}_{y}}{\mathrm{e}_{z}} & 0 \\
0 & 0 & 1 & 0 \\
0 & 0 & 0 & 1
\end{array}\right]=\left[\begin{array}{llll}
1 & 0 & 0 & 0 \\
0 & 1 & 0 & 0 \\
0 & 0 & 1 & 0 \\
0 & 0 & 0 & 1
\end{array}\right]
$$

Where $\mathrm{e}_{\mathrm{x}}=0, \mathrm{e}_{\mathrm{y}}=0$ and $\mathrm{e}_{\mathrm{z}}=-\rho$ are $\mathrm{X}, \mathrm{Y}$ and $\mathrm{Z}$ coordinates of the tube focus in the object space. The perspective scaling transformation is $\mathrm{M}_{\mathrm{S}}$ as the following,

$$
\mathrm{M}_{\mathrm{S}}=\left[\begin{array}{cccc}
1 & 0 & 0 & 0 \\
0 & 1 & 0 & 0 \\
0 & 0 & 1 & 0 \\
0 & 0 & -\frac{1}{\mathrm{e}_{z}} & 1
\end{array}\right]=\left[\begin{array}{cccc}
1 & 0 & 0 & 0 \\
0 & 1 & 0 & 0 \\
0 & 0 & 1 & 0 \\
0 & 0 & \frac{1}{\rho} & 1
\end{array}\right]
$$

Where $\rho$ is the distance between the tube focus and the image plane. Thus the shear transformation matrix $\mathrm{M}_{\text {shear }}$ can be described as: 


$$
\mathrm{M}_{\text {shear }}=\mathrm{M}_{\mathrm{s}} \cdot \mathrm{M}_{\mathrm{sh}}=\left[\begin{array}{cccc}
1 & 0 & -\frac{\mathrm{e}_{\mathrm{x}}}{\mathrm{e}_{z}} & 0 \\
0 & 1 & -\frac{\mathrm{e}_{\mathrm{y}}}{\mathrm{e}_{\mathrm{z}}} & 0 \\
0 & 0 & 1 & 0 \\
0 & 0 & -\frac{1}{\mathrm{e}_{\mathrm{z}}} & 1
\end{array}\right]=\left[\begin{array}{cccc}
1 & 0 & 0 & 0 \\
0 & 1 & 0 & 0 \\
0 & 0 & 1 & 0 \\
0 & 0 & \frac{1}{\rho} & 1
\end{array}\right]
$$

The warp transformation is the product of the view transformation matrix and the inverse of the shear transformation matrix.

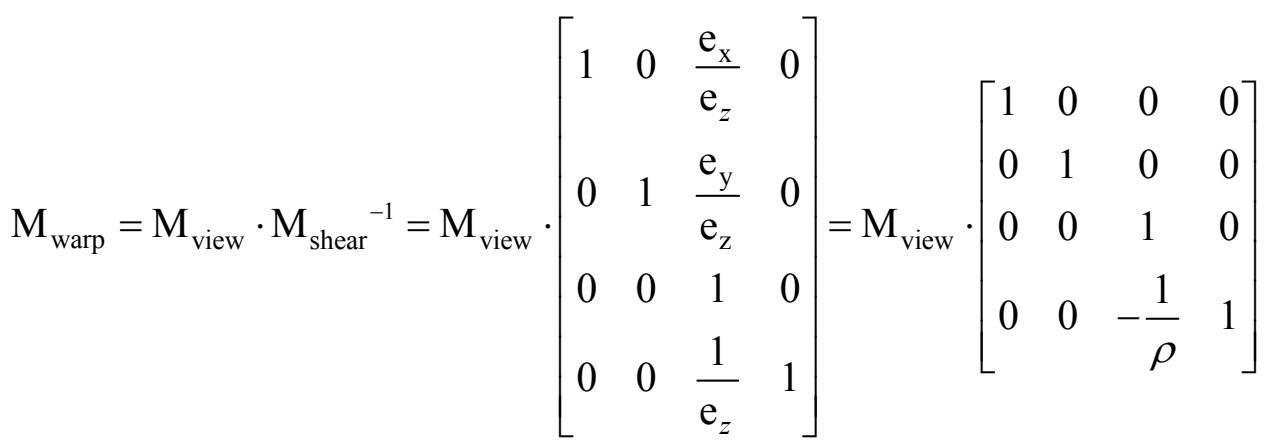

We use the projection parameters that were used for real DEDR image acquisitions. The parameters include the distance between the X-ray tube's focus and the detector plane. We determine the location of the X-ray tube and the principal view axis. These parameters are used to compute the perspective transformation matrix. Based the shear-warp factorization method, we developed Gaussian weighted projection using a Gaussian function as the weighting function during the projection processing.

\subsection{Gaussian Weighted Projection Methods}

Gaussian distribution as a model of quantitative phenomena in the natural and behavioral sciences is due to the central limit theorem. Many measurements and physical phenomena (like photon counts and noise) can be approximated well by the Gaussian distribution [12]. While the mechanisms underlying these phenomena are often unknown, the use of the Gaussian distribution model can be theoretically justified by assuming that many small, independent effects are additively contributing to each observation.

To indicate that a random variable $X$ is normally distributed with a mean of $\mu$ and a variance of $\sigma^{2}$, we write $X \sim N$ $\left(\mu, \sigma^{2}\right)$. The probability density function of the Gaussian distribution is a Gaussian function,

$$
\mathrm{f}(x: \mu, \sigma)=\frac{1}{\sigma \sqrt{2 \pi}} \mathrm{e}^{-\frac{1}{2}\left(\frac{x-\mu}{\sigma}\right)^{2}}=\frac{1}{\sigma} \varphi\left(\frac{x-\mu}{\sigma}\right)
$$

Where $\sigma$ is the standard deviation, and, $\mu$ is the expected value, and

$$
\varphi(x)=\frac{1}{\sqrt{2 \pi}} \mathrm{e}^{-\frac{1}{2} x^{2}}
$$

is the density function of the "standard" normal distribution.

We assume that CT intensity values of calcified tissue have a Gaussian distribution and that CT intensity values of all tissue can modeled as one or more Gaussian distributions.

$$
\mathrm{X}=\sum_{1}^{\mathrm{n}} \mathrm{N}\left(\mu_{\mathrm{n}}, \sigma_{\mathrm{n}}\right)
$$

In addition, the sum of various Gaussian distributions is also Gaussian distributed with: 


$$
\mathrm{U}=\mathrm{X}+\mathrm{Y} \sim \mathrm{N}\left(\mu \mathrm{X}+\mu \mathrm{Y}, \sigma_{\mathrm{X}}^{2}+\sigma_{\mathrm{Y}}^{2}\right)
$$

Actually, the accumulation of calcification in the coronary arteries is a process similar to new bone formation [2]. Histological studies support the association of tissue densities of $130 \mathrm{HU}$ with calcified plaque [1]. The CT values within a calcification region can be described as a series of variables with an approximate Gaussian distribution. The image intensities within this region are described as $\mathrm{I} \sim \mathrm{N}\left(\mu, \sigma^{2}\right)$.

In our projection method, we use a Gaussian function, which is same with the calcification distribution, to weight the volume. That will enhance the voxel within the calcification distribution and limit other voxels outside of the distribution. Thereby, we can highlight the calcification from the heart or body voxels. The weighted projection factor is a Gaussian function as follows:

$$
\operatorname{Weight}(I)=\mathrm{e}^{-\frac{1}{2}\left(\frac{1-\mu}{\sigma}\right)^{2}}
$$

During the Shear-warp factorization processing, the $\mathrm{M}_{\text {view }}$ matrix becomes:

$$
\mathrm{M}_{\text {view }}=\mathrm{M}_{\text {warp }} \cdot \mathrm{M}_{\text {shear }} \cdot \text { Weight }
$$

To selectively reconstruct and project a calcification region, we use a Gaussian function as the weighting factor during the projection processing. The projection result depends on the mean $\mu$ and the standard deviation $\sigma$.

\section{EXPERIMENTS}

\subsection{Digital Phantom Experiments}

We used simulated digital phantoms to verify the selectivity of Gaussian Weighted Projection method. The phantom includes three objects and their intensity distributions meet three Gaussian distributions, i.e. $\mathrm{N}(50,30), \mathrm{N}(450,280)$ and $\mathrm{N}(1025,65)$. The objects are to simulate soft tissue, calcium, and bone tissue, respectively. We use multiple Gaussian functions to fit the histogram of the digital phantom, and then generated DRR images using the mean and standard deviation parameters of the fitting results. We compared the DRR images with the ground truth to evaluate if the Gaussian Weighted Projection can differentiate the three objects, respectively.

\subsection{Physical Phantom Experiment}

We designed a physical phantom with fiducial markers to evaluate the selectivity of the Gaussian Weighted Projection method. As shown in Figure 2 (Right), we first developed a physical phantom that included 101 different concentrations of $\mathrm{Ca}\left(\mathrm{H}_{2} \mathrm{PO} 4\right)_{2}$ samples from $0.25 \mathrm{ug} / \mathrm{ml}$ to $0.25 \mathrm{mg} / \mathrm{ml}$ with a step concentration $2.5 \mathrm{ug} / \mathrm{ml}$ and an addition blank sample as the reference. All samples were sealed and put into a plastic tube. Nine fiducial markers were applied to the plastic tube to assist identification of the sample position.

We acquired micro-CT images from physical phantom using a micro-CT system (X-SPECT ${ }^{\text {, }}$, Gama Medica, Northridge, $\mathrm{CA}$ ) at the $45-\mathrm{KVp} 750$-microAmp energy level. The resolution of the micro-CT images is $0.1 \mathrm{~mm}$. The image was acquired by size $512 \times 512 \times 512$. To avoid the affect of air bubble or other materials, we select middle portion of the images for the subsequent processing, i.e. histogram calculation and Gaussian function curve fitting. We generated DRR images using the mean and standard deviation parameters of the fitting results and then evaluate the DRR images.

\subsection{Clinical volume experiment}

We identified a group of 12 clinical patients with findings suspicious for cardiac calcification on the dual-energy digital radiography (DEDR) images. These patients had undergone multi-detector CT (MDCT) evaluation of the chest within 12 months of chest X-ray digital radiography. The CT examinations were obtained for a variety of clinical indications using imaging protocols that varied considerably in slice thickness, radiographic technique, and presence or absence of intravenous (IV) contrast material. The CT studies were analyzed for the presence of coronary artery, valvular, or myocardial calcification. 
We extracted the heart from one clinical chest CT volume and then manually segmented coronary calcification to do the histogram processing and Gaussian function curve fitting. The heart volume is a cube between the aortic arch and the diaphragm, including all chambers of the heart. The volume size is $200 \times 200 \times 160$. The image volume of the segmented coronary calcification is the same size of the heart volume, while only containing the calcification. We generated DRR images using the mean and standard deviation parameters of the fitting results and then examined the DRR images.

To compare the visualization effect, we used the heart volume to perform projections using the AVG, MIP and GWP methods. Each method does projection at 190 degrees and 280 degrees from the posterior to anterior direction. . We used the manually segmented calcium as the reference to evaluate the visualization from the three projection methods.

\section{RESULTS}

Figure 1 shows the histogram of the digital phantom. The curve fitting results in three Gaussian functions, i.e. $\mathrm{N}(49.54,29.92), \mathrm{N}(449.34,283.36)$ and $\mathrm{N}(1024.66,64.75)$. Compared to the ground truth $\mathrm{N}(450,280), \mathrm{N}(50,30)$ and $\mathrm{N}(1025,65)$, the fitting results are excellent. The projection images show that the Gaussian-weighted projection algorithm can selectively display each type of simulated tissue, which was not achievable by the MIP and AVG methods.

Figure 2 shows the histogram of the physical phantom, which was fitted with five Gaussian functions. They were related with noise, the plastic tube, calcification crystal, the glass tubes and calcification solution. Figure 3 shows the projection images of the physical phantom using the three projection methods. The Gaussian weighted project method can selectively display each material.

Figure 4 shows the histogram of the heart CT images. The curve fitting resulted in two Gaussian functions: $\mathrm{N}(34.77$, 18.51) and $\mathrm{N}(-27.00,49.01)$. Based on the projection images and the range of the parameters, we believe that first function is related with the myocardial and blood and that the second is related with the fat tissue.

Figure 5 shows the histogram of the segmented calcification volume. Three Gaussian functions, i.e. N(-21.90, 45.23), $\mathrm{N}(59.78,124.19)$ and $\mathrm{N}(302.76,228.15)$, are related to the fat tissue, fabric tissue and calcium, respectively. Compared to the AVG and MIP image, the GWP method can display each type of real tissue selectively. The distribution of the calciumcan be modeled as $\mathrm{N}(302.76,228.15)$ in this patient.

Figure 6 shows the visual comparison among the AVG, MIP and GWP methods for the heart. The GWP method used the parameters $\mathrm{N}(302.76,228.15)$, which were derived from the segmented calcium. The GWP image demonstrated that the calcified tissue presents as the tortuous thin and long slices. But it is difficult to visualize the tissue by either AVG or MIP. The GWP better visualizes the calcium than the MIP and AVG methods.

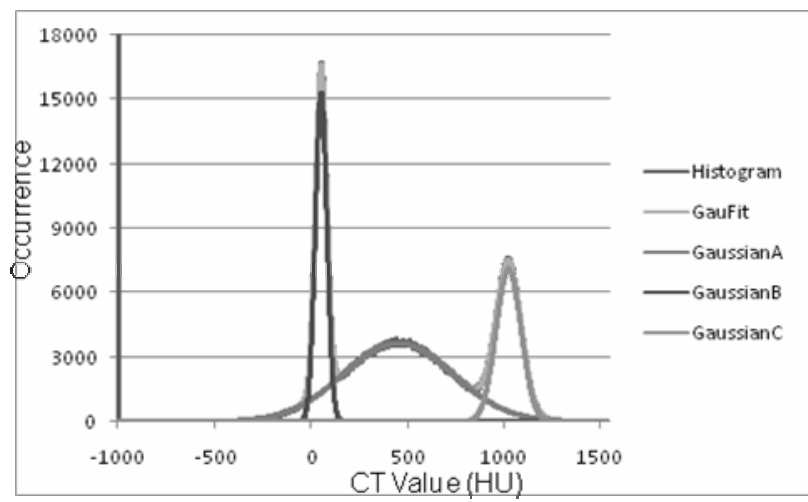

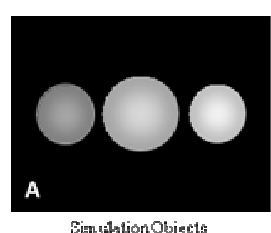

Bimantistionobjects

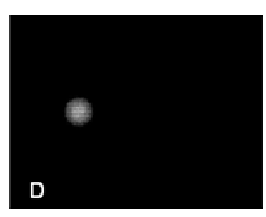

H(d9 S4.29 92)

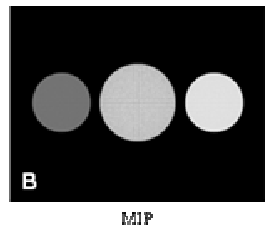

MIP

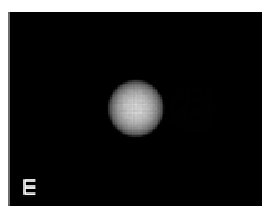

$\mathrm{N}(44934,2 \mathrm{~B}] 36)$

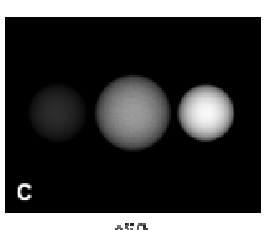

AVO

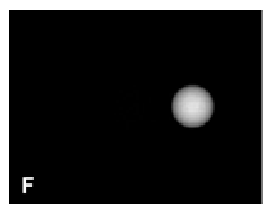

M. L024

Figure 1: Left: The histogram of the digital phantom. Right: Gaussian weighted projection for the digital phantom. The phantom consists of three simulated tissues that have the intensity distributions of $\mathrm{N}(450,280), \mathrm{N}(50,30)$ and $\mathrm{N}(1025,65)$, respectively. The three Gaussian functions, i.e. $\mathrm{N}(49.54,29.92), \mathrm{N}(449.34,283.36)$ and $\mathrm{N}(1024.66,64.75)$, well fit the histogram. As computed to the original objects (A), the MIP image (B) and the AVG image cannot selectively display any one of the three objects. However, the Gaussian-weighted projection method can display each of the three objects using different Gaussian functions. 

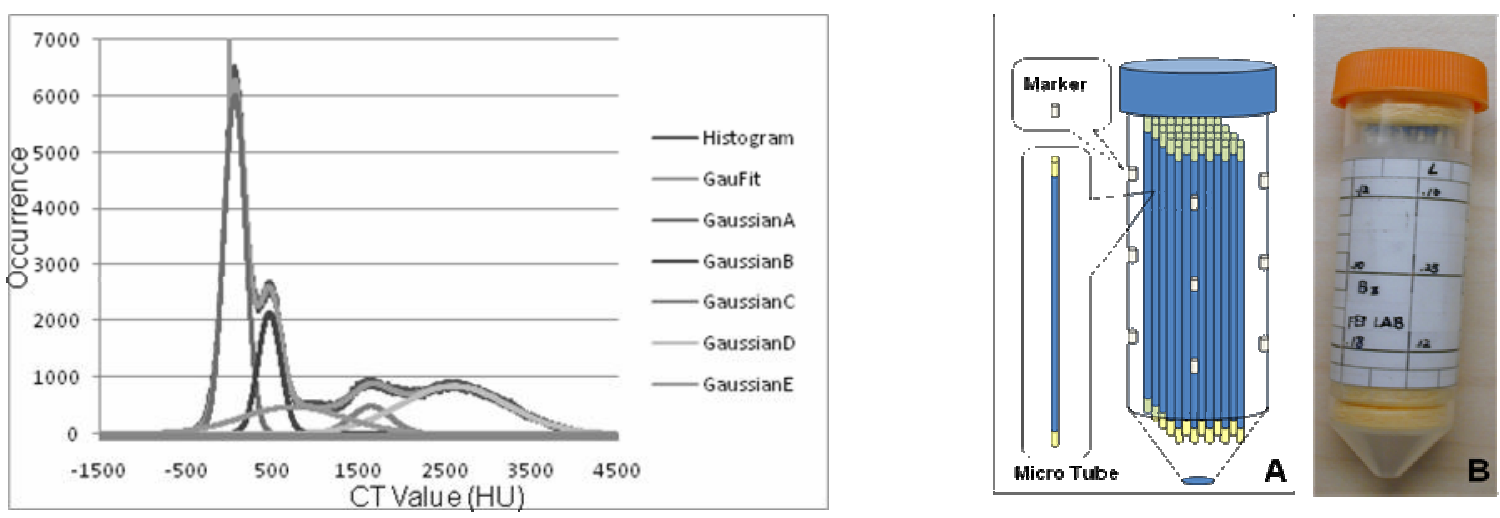

Figure 2: Left: Histogram of the physical phantom. Right: Pictures of the physical phantom. The draw of the phantom (A) show the micro-tubes and the nine markers. $\mathrm{B}$ is the picture of phantom with the markers.
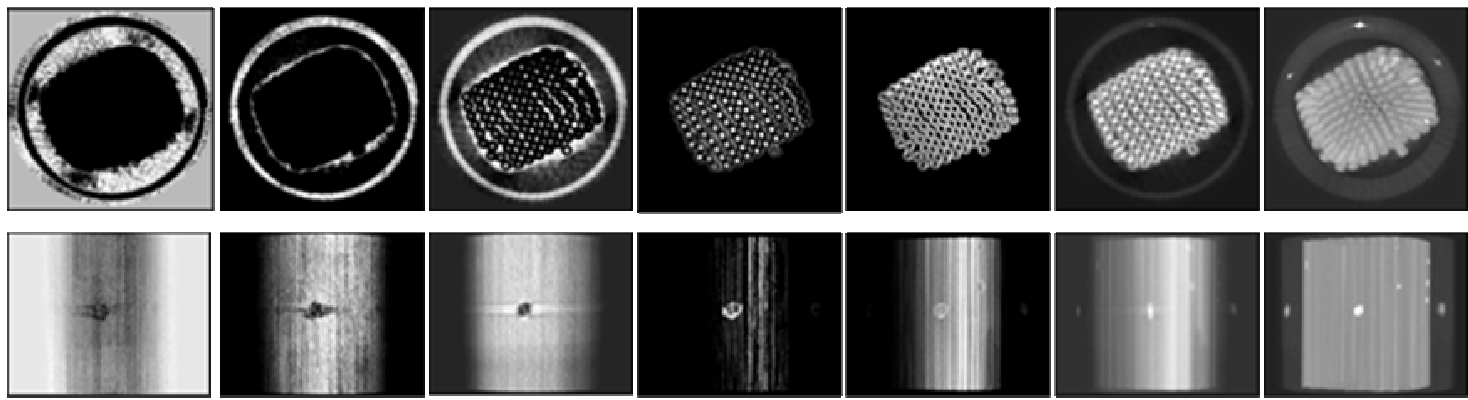

Gaussian A: Gaussian B:
Noise
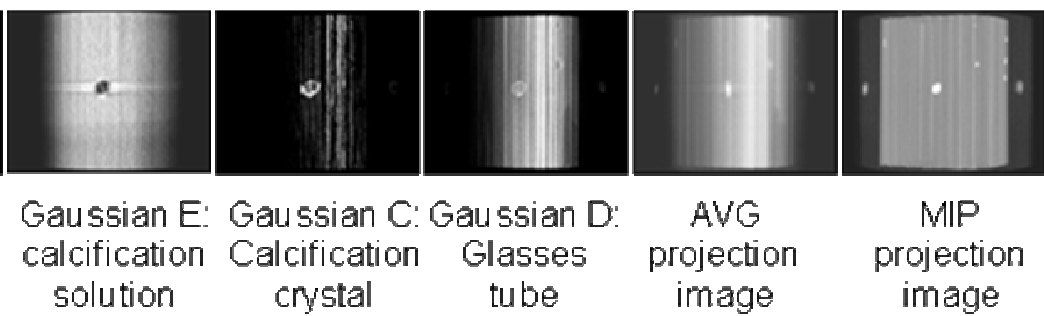

AVG projection image

MIP projection image

Figure 3: Gaussian weighted projection for the physical phantom. The histogram was fitted with five Gaussian functions that represent different materials. A: $\mathrm{N}(60.21,124.76)$ is the background noise; $\mathrm{B}: \mathrm{N}(460.35,131.22)$ is the plastic tube; C: $\mathrm{N}(1636.03,230.22)$ is calcification solution; $\mathrm{D}: \mathrm{N}(2595.09,619.07)$ is the crystal of the calcification; and E: $\mathrm{N}(732.41,593.39)$ is the glass tubes. It is possible to differentiate different materials using the Gaussian-weighted projection method. The AVG and MIP cannot selectively display each material.

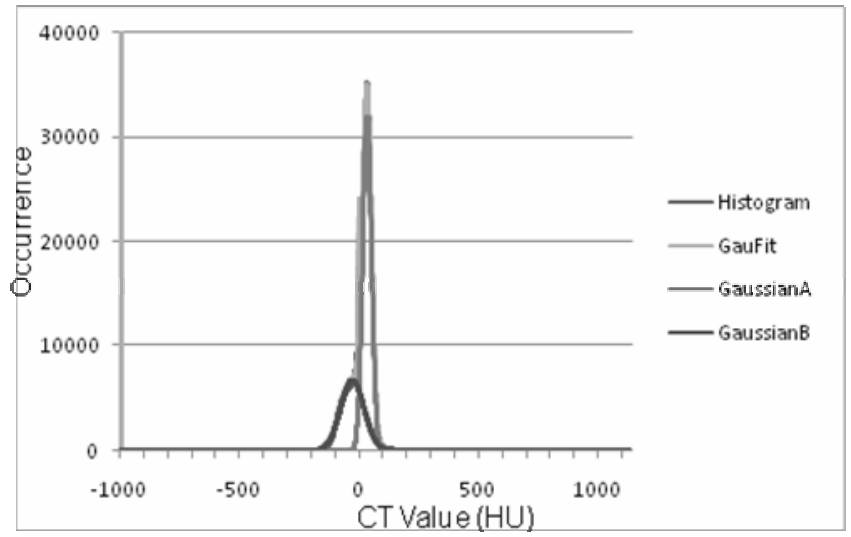

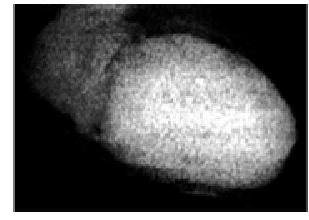

Sal ssian myncarcilal and bilond

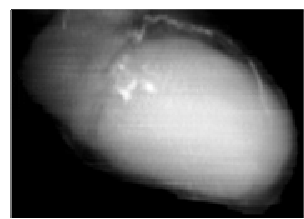

AVG projection image

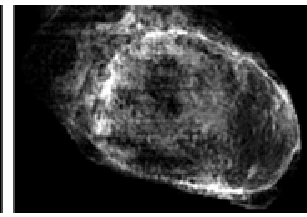

Gausian $B$ fallissue

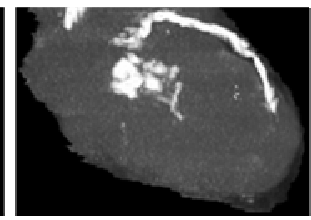

MIP projection Image

Figure 4: Left: Histogram of the heart. Right: Gaussian weighted projection for the heart.. Gaussian function A N(34.77, $18.51)$ is the myocardial and blood. Gaussian function B N(-27.00, 49.01) is the fat tissue outside of the pericardium. The AVG and MIP images are shown below for comparison. 

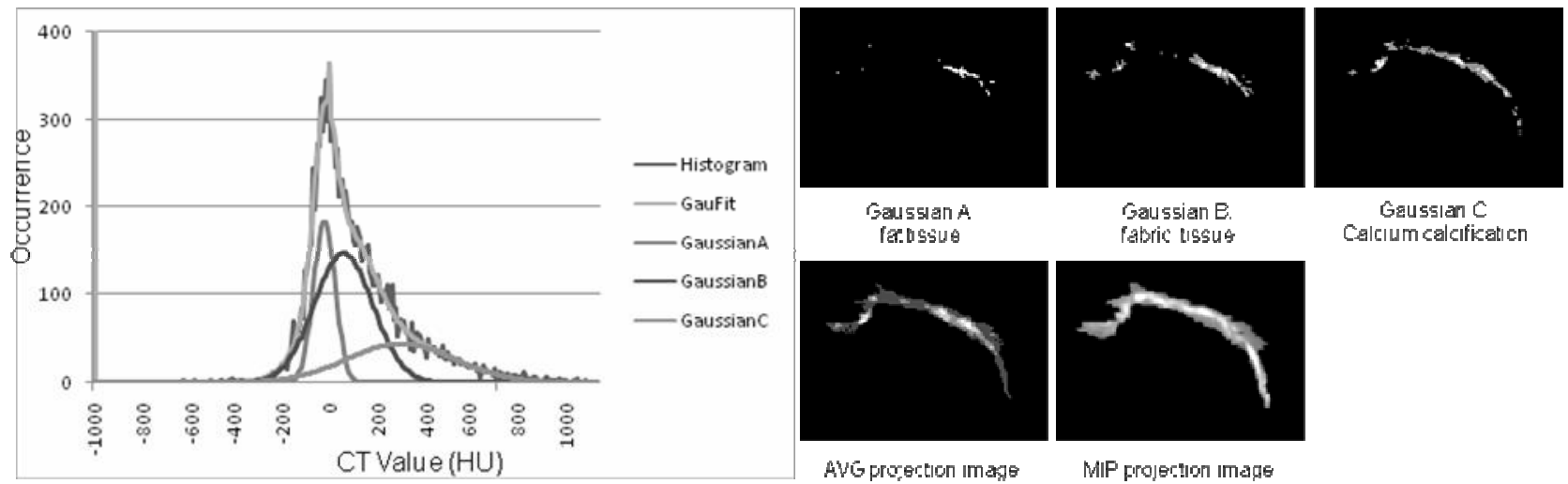

Saussian $\mathrm{B}$. rabric lissug

Gaussian C Calcium calcificaticn

Figure 5: Left: Histogram of the segmented calcification. Right: Gaussian weighted projection for the segmented calcification. Gaussian function A N $(-21.90,45.23)$ is the fat tissue. Gaussian function B N $(59.78,124.19)$ is the the fabric tissue.Gaussian function $\mathrm{C} \mathrm{N}(302.76,228.15)$ is the calcium. The AVG and MIP images are shown below for comparison.
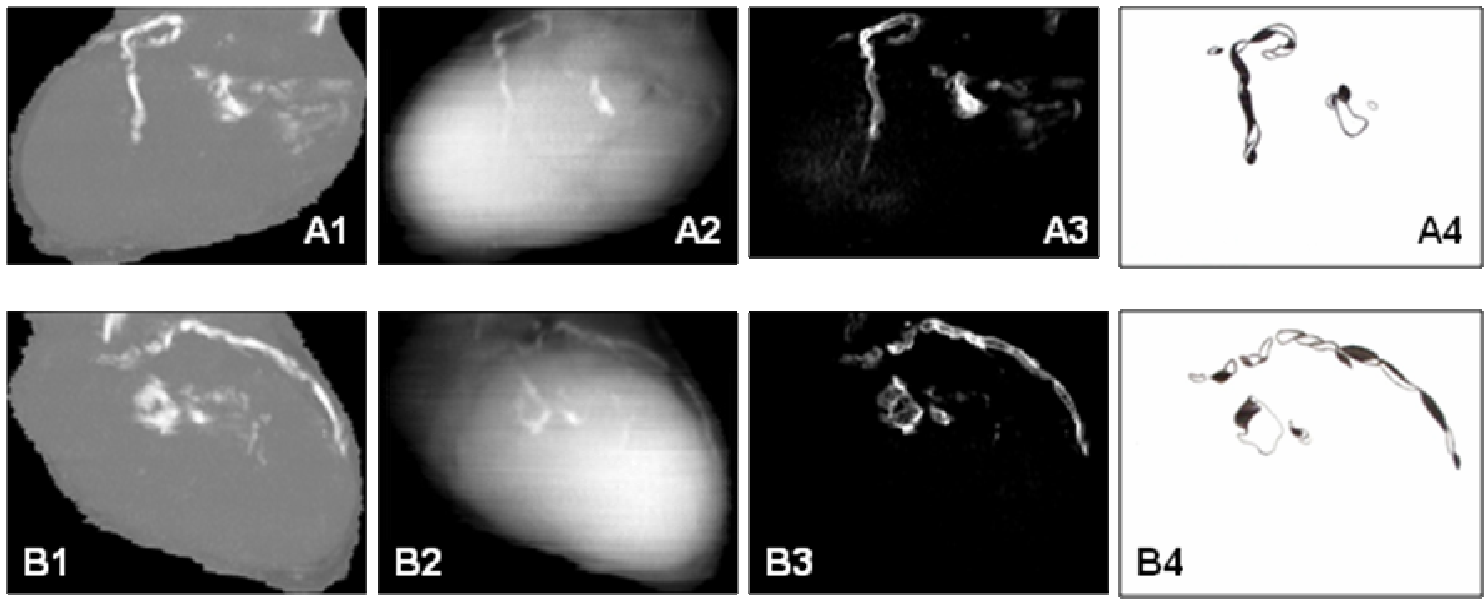

Figure 6: Visualization of calcified coronary artery. Top:A1-A4 are the projection images at 190 degrees from the PA direction. Bottom: B1-B4 are the projection images at 280 degrees. A1 and B1 are the MIP images; A2 and B2 are the AVG images; A3 and B3 are the GWP images; and A4 and B4 are the manual segmentation and visualization of the coronary artery calcification. The calcium presents as the tortuous thin and long slices. GWP visualizes the calcium better than MIP and AVG methods.

\section{CONCLUSIONS}

We have developed a Gaussian weighted projection method for the visualization of cardiac calcification. Our digital and physical phantom studies show that the GWP method can selectively display different type of tissues. Clinical images show that the Gaussian-weighted projection method better visualizes cardiac calcification than either AVG or MIP. This GWP projection method can be used to evaluate DEDR as a screening tool for the detection of coronary artery diseases. The visualization method can also have other applications such as image-guided catheterization for cardiac interventions. 


\section{ACKNOWLEDGEMENTS}

The algorithm developed in this research was partially supported by the NIH grant R21CA120536 (PI: Fei), the Presidential Initiative Award of Case Western Reserve University (PI: Fei), and the Case Comprehensive Cancer Center Pilot Award (PI: Fei). XC was partially supported by the CSC Scholarship and the Case Center for Imaging Research which is supported by the NIH/NCI R24 grant (CA110943). The authors thank Elena DuPont for the clinical CT and DR image collections, Yuguo Li and Alison Hao for physical phantom making and data acquisitions, and Dr. Hanping $\mathrm{Wu}$ for the visual inspection.

\section{REFERENCES}

1. B.H.Thompson and W.Stanford, "Imaging of coronary calcification by computed tomography," J.Magn Reson.Imaging, 19, 720-733( 2004).

2. L.Wexler, B.Brundage, J.Crouse, R.Detrano, V.Fuster, J.Maddahi, J.Rumberger, W.Stanford, R.White, K. Taubert, "Coronary artery calcification: pathophysiology, epidemiology, imaging methods, and clinical implications. A statement for health professionals from the American Heart Association. Writing Group," Circulation, 94, 1175-1192( 1996)

3. P. Greenland, R. Bonow, B. Brundage, et.al. " ACCF/AHA 2007 Clinical Expert Consensus Document on Coronary Artery Calcium Scoring by Computed Tomography in Global Cardiovascular Risk Assessment and in Evaluation of Patients With Chest Pain: a report of the American College of Cardiology Foundation Clinical Expert Consensus Task Force (ACCF/AHA Writing Committee to Update the 2000 Expert Consensus Document on Electron-Beam Computed Tomography)" Circulation, 115, 402-426, (2007)

4. R.C.Gilkeson, R.D.Novak, P.Sechs, "Digital radiography with dual-energy subtraction: improved evaluation of cardiac calcification," AJR Am Roentgenol. 183( 5), 1233-1238( 2004).

5. B.H.Thompson and W.Stanford, "Update on using coronary calcium screening by computed tomography to measure risk for coronary heart disease," Int.J.Cardiovasc.Imaging, 21, 39-53( 2005).

6. C.Schaefer-Prokop, M.Uffmann, E.Eisenhuber, M.Prokop. "Digital radiography of the chest: detector techniques and performance parameters," J.Thorac.Imaging, 18, 124-137( 2003).

7. C.P.Heussel, T.Voigtlaender and H.Kauczor etc. "Detection of coronary artery calcifications predicting coronary heart disease: comparison of fluoroscopy and spiral CT," Eur.Radiol., 8, 1016-1024( 1998).

8. H.MacMahon, "Digital chest radiography: practical issues," J.Thorac.Imaging, 18, 138-147( 2003).

9. B.Fei, X.Chen, H.Wang, et.al., "Automatic Registration of CT Volumes and Dual-Energy Digital Radiography for Detection of Cardiac and Lung Diseases," Proceedings of the 28th Annual Conference of IEEE Engineering in Biology and Medicine , New York USA, 1976-1979( 2006).

10. X.Chen, R.C.Gilkeson, and B.Fei, "Automatic intensity-based 3D-to-2D registration of CT volume and dual-energy digital radiography for the detection of cardiac calcification," Proceedings of the SPIE medical imaging, 6512, 65120A,( 2007)

11. P.G.Lacroute, "Fast Volume Rendering Using A Shear-Warp Factorization Of The Viewing Transformation," Anonymous Technical Report: CSL-TR- 95-678, Computer Systems Laboratory, Departments of Electrical Engineering and Computer Science. Stanford, CA. Stanford University, (1995)

12. "Normal distribution," http://en.wikipedia.org/wiki/Normal_distribution, Wikipedla, The Free Encyclopedia. 
Xiang Chen, Ke Li, Robert Gilkeson, and Baowei Fei, "Gaussian weighted projection for visualization of cardiac calcification”, Michael I. Miga, Kevin Robert Cleary, Proc. SPIE 6918, 691831 (2008)

Copyright 2008 Society of Photo-Optical Instrumentation Engineers (SPIE). One print or electronic copy may be made for personal use only. Systematic reproduction and distribution, duplication of any material in this paper for a fee or for commercial purposes, or modification of the content of the paper are prohibited.

http://dx.doi.org/10.1117/12.772597 